\title{
Association between PCSK9 Levels and Markers of Inflammation, Oxidative Stress, and Endothelial Dysfunction in a Population of Nondialysis Chronic Kidney Disease Patients
}

\author{
Evangelia Dounousi $\mathbb{D}^{1},{ }^{1}$ Constantinos Tellis $\left(\mathbb{D},{ }^{2}\right.$ Paraskevi Pavlakou $\left(\mathbb{D},{ }^{3}\right.$ Anila Duni $\mathbb{D},{ }^{1}$ \\ Vasillios Liakopoulos $₫$, ${ }^{4}$ Patrick B. Mark $\left(1,{ }^{5}\right.$ Aikaterini Papagianni $\odot{ }^{6},{ }^{6}$ \\ and Alexandros D. Tselepis $\mathbb{D}^{2}$ \\ ${ }^{1}$ Department of Nephrology, School of Health Sciences, University of Ioannina, Ioannina, Greece \\ ${ }^{2}$ Atherothrombosis Research Centre/Laboratory of Biochemistry, Department of Chemistry, University of Ioannina, Ioannina, Greece \\ ${ }^{3}$ Department of Nephrology, University Hospital of Patras, Patras, Greece \\ ${ }^{4}$ Division of Nephrology and Hypertension, 1st Department of Internal Medicine, AHEPA Hospital, School of Medicine, \\ Aristotle University of Thessaloniki, Greece \\ ${ }^{5}$ Institute of Cardiovascular and Medical Sciences, University of Glasgow, Glasgow, UK \\ ${ }^{6}$ Department of Nephrology, School of Medicine, Aristotle University of Thessaloniki, Hippokration Hospital, Thessaloniki, Greece
}

Correspondence should be addressed to Evangelia Dounousi; evangeldou@gmail.com

Received 13 December 2020; Accepted 16 June 2021; Published 21 July 2021

Academic Editor: Luciana Mosca

Copyright (C) 2021 Evangelia Dounousi et al. This is an open access article distributed under the Creative Commons Attribution License, which permits unrestricted use, distribution, and reproduction in any medium, provided the original work is properly cited.

\footnotetext{
Proprotein convertase subtilisin/kexin 9 (PCSK9) plays an important role in lipid metabolism while available literature regarding its involvement in the pathogenesis of atherosclerosis and in the expression of genes associated with apoptosis and inflammation is constantly increasing. Patients with chronic kidney disease (CKD) experience disproportionately increased cardiovascular morbidity and mortality due to dyslipidemia, accelerated atherosclerosis, inflammation, oxidative stress, and other risk factors. In the present cross-sectional study, we investigated the possible association of serum PCSK9 levels with markers of inflammation, oxidative stress, and endothelial damage in patients with CKD. Patients and Methods. Ninety-two patients with CKD stages II-IV (eGFR CKD-EPI $47.3 \pm 25.7 \mathrm{ml} / \mathrm{min} / 1.73 \mathrm{~m}^{2}$, mean age 66 years, $51 \mathrm{men}$ ) were included in the study. Plasma PCSK9 levels were correlated with comorbidities (arterial hypertension, diabetes mellitus, and history of cardiovascular disease), renal function indices (eGFR, proteinuria-UPR/24h), lipid parameters (LDL-cholesterol, HDL-cholesterol, triglycerides, Lp(a), APO-A1, and APO-B), and soluble biomarkers of inflammation, oxidative stress, and endothelial damage (hs-CRP, fibrinogen, 8-epiPGF2a, ox-LDL, IL-6, TNF- $\alpha$, sICAM-1, and sVCAM-1). Results. The mean plasma value of PCSK9 was $278.1 \mathrm{ng} / \mathrm{ml}$. PCSK9 levels showed direct correlation with serum triglycerides $(p=0.03), \operatorname{Lp}(\mathrm{a})(p=0.01)$, and sICAM-1 levels $(p=0.03)$. There was no significant correlation between PCSK9 levels and indices of the renal function, other lipid profile parameters, inflammatory markers, or comorbidities. Multiple regression analysis showed a significant effect of Lp(a) on PCSK9 levels, and for each unit of higher $\mathrm{Lp}(\mathrm{a})$, an increase by 3.082 is expected (95\% CI: $0.935-5.228, p=0.006)$. At the same time, patients receiving statins are expected to have on average $63.8 \mathrm{ng} / \mathrm{ml}$ higher PCSK9 values compared to patients not receiving statins (95\% CI: 14.6-113.5, $p=0.012$ ). Conclusion. Plasma levels of PCSK9 in nondialysis CKD patients are correlated with endothelial dysfunction and lipid metabolism parameters. Statin intake increases PCSK9 levels significantly in this patient population. PCSK9 levels are not correlated with the severity of kidney disease. Major prospective studies are necessary to investigate the role of PCSK9 in the atherosclerotic cardiovascular outcome in CKD.
} 


\section{Introduction}

Chronic kidney disease (CKD) has been characterized as an atherosclerosis multiplier increasing disproportionally the incidence of fatal and nonfatal cardiovascular events from the early stages [1]. Almost a quarter of patients with mild to moderate $\mathrm{CKD}$ have been reported to die mainly due to atherosclerotic cardiovascular disease (ASCVD) before the initiation of renal replacement therapy while non-ASCVD becomes dominant at more advanced stages of CKD [2, 3]. Atherosclerosis, beyond being a lipid disorder, is characterized by major inflammatory properties and has been implicated in the pathogenesis of arterial plaque formation and rupture and in clinical outcomes $[4,5]$. Chronic inflammation, oxidative stress, and endothelial dysfunction separately and through their crosstalk have direct implications in the pathogenesis of atherosclerosis in CKD patients and have been established as nontraditional cardiovascular risk factors in this frail population [6-9].

Dyslipidemia, on the other hand, is one of the traditional cardiovascular risk factors. Lipid metabolism dysregulations are well recognized in patients with CKD $[10,11]$. The common pattern of lipid disorders in a CKD patient consists of increased levels of triglycerides (TG) and lipoprotein (a) $(\mathrm{Lp}(\mathrm{a}))$, reduced levels of high-density lipoprotein cholesterol (HDL-C), and normal or slightly reduced total cholesterol (T-Chol) and low-density lipoprotein cholesterol (LDL-C) $[10,11]$. Proprotein convertase subtilisin/kexin type 9 (PCSK9) has been identified as a central regulator of the LDL-receptor (LDL-R) expression by binding on the hepatocyte LDL-R, causing a reduction in the number of LDL-R and a subsequent enhancement in circulating LDL-C levels [12]. In CKD patients, existing literature is conflicting about possible association of PCSK9 plasma levels with the renal function. In the largest published observational study in two independent cohorts of nondialysis patients, Rogacev et al. showed no association of PCSK9 with renal function or with cardiovascular mortality [13]. Monoclonal antibodies against PCSK9 (PCSK9i) have emerged as safe and efficient hypolipidemic agents. In large clinical trials, PCSK9i manage to reduce LDL-C to target levels and Lp(a) levels by $20-30 \%$ with additional cardiovascular benefit when added to statin treatment in high-risk populations [14]. Nevertheless, efficacy of the PCSK9i alirocumab in CKD stage 3 patients was estimated in an analysis that pooled data from eight phase III ODYSSEY trials showing an efficacy and safety profile similar to that of patients with preserved eGFR [15].

Beyond the key role of PCSK9 on lipid disorders and management, emerging evidence points out its determining implication in the inflammatory arm of atherosclerosis. Experimental data have shown the upregulatory effect of proinflammatory and oxidative stress factors on the PCSK9 expression and vice versa, accordingly the possible modulatory role of PCSK9 on inflammation mediators [16]. Tumor necrosis factor alpha (TNF- $\alpha$ ) resulted in the induction of PCSK9 mRNA and protein expression in HepG2 cells and vascular smooth muscle cells (VSMCs) [17], while oxidized low-density lipoprotein (ox-LDL) upregulated the PCSK9 expression in different cells, such as vascular endothelial cells
(ECs), VSMCs, macrophages, and dendritic cells [18-23]. The administration of lipopolysaccharide in a PCSK9 knockout mice $\left(\mathrm{PCSK}^{-/-}\right.$) reduced plasma levels of TNF- $\alpha$ and interleukin 6 and 10 (IL-6, IL-10) [24], whereas knockdown of PCSK9 inhibited the inflammatory response in macrophages promoted by ox-LDL [19]. Moreover, PCSK9 has been implicated in endothelial dysfunction by upregulating the expression of intercellular adhesion molecule-1 (ICAM-1) and vascular cell adhesion molecule-1 (VCAM-1) in endothelial cells [16].

Experimental results are supported only by a limited number of clinical studies investigating the putative role of PCSK9 in the inflammatory process. Plasma levels of inflammatory cytokines, TNF- $\alpha$, IL- 6 , and IL- 8 were decreased in septic patients with a PCSK9 loss of function allele [24] while patients with bacteremia had increased PCSK9 plasma levels in a direct association with CRP [25]. Moreover, CRP as a marker of inflammation and ASCVD has been positively associated with plasma levels of PCSK9 both in patients with acute coronary syndromes and stable coronary disease [26]. In the same line, fibrinogen levels have been associated with PCSK9 levels in patients with stable coronary disease independently of traditional cardiovascular risk confounding [27].

Despite the emerging role of PCSK9 in ASCVD [28], there is lack of robust clinical data investigating the interplay of PCSK9 with markers of inflammation, oxidative stress, and endothelial dysfunction in high-risk patients such as CKD patients. Having this in mind, we designed a cross-sectional, observational study to investigate possible association of PCSK9 with inflammation markers, including highsensitivity C-reactive protein (hs-CRP), IL-6, TNF- $\alpha$, oxidative stress markers, 8 -isoprostanes (8-epiPGF2a), and oxLDL, and endothelial dysfunction markers such as (sICAM1) and (sVCAM-1) in a nondialysis population of stable CKD patients. As a secondary outcome, we investigated the possible association of PCSK9 levels with renal function markers, lipid metabolism markers, and echocardiographic indices.

\section{Patients and Methods}

2.1. Participants. Ninety-two stable, CKD stage II- $I \mathrm{~V}$ adults from the outpatient clinics of the Nephrology Department of our Tertiary Hospital were included in this single-center, cross-sectional, observational study. Twenty healthy volunteers attending the Outpatient Obesity and Lipid Clinic of the University Hospital of Ioannina, Greece, were recruited for the study in order to serve as a control group. The exclusion criteria were a recent major cardiovascular event (within the past 3 months before recruitment), active infection or history of infection in the last month, severe heart failure (NYHA IV) and/or severe valvulopathy, hepatic cirrhosis, active malignancy, and current immunosuppression treatment. All participants after being informed in detail provided signed informed consent to participate in the study. The study was approved by the Scientific Committee of the University Hospital of Ioannina, Greece, while all requirements of Helsinki Declaration were met. 
2.2. Anthropometric Variables-Biochemical Parameters. On recruitment, all patients underwent a detailed review of their medical history and a careful clinical examination. The protocol of the study included recording demographic characteristics, smoking habits and alcohol consumption, primary renal disease, history of comorbidity and medication, anthropometric measurements, and measurement of blood pressure and heart rate. A routine full hematologic and biochemical screening was performed. All laboratory measurements were carried out after an overnight fast while water consumption was allowed. Plasma (using EDTA as an anticoagulant) and serum were prepared from blood samples and stored in aliquots at $-80^{\circ} \mathrm{C}$. Lipid profile assessment included serum levels of T-Chol, TC, and HDL-C that were determined enzymatically on an Olympus AU600 clinical chemistry analyzer (Olympus Diagnostica, Hamburg, Germany), LDL-C was calculated using the Friedewald formula, and $\mathrm{Lp}(\mathrm{a})$, apolipoprotein A1 (APO-A1), and apolipoprotein B (APO-B) were measured with a Behring Holding $\mathrm{GmbH}$ analyzer (Liederbach, Germany). All the above measurements were conducted in the laboratory facilities of the University Hospital of Ioannina. For the calculation of the estimated glomerular filtration rate (eGFR, $\mathrm{ml} / \mathrm{min} / 1.73 \mathrm{~m}^{2}$ ), the Chronic Kidney Disease Epidemiology Collaboration (CKD-EPI) equation was used [29]. Proteinuria was estimated in a 24 -hour urine collection (UPR, mg/24h).

2.3. Determination of PCSK9. Plasma PCSK9 concentration was determined by a quantitative sandwich enzyme immunosorbent assay using a commercially available kit according to the instructions provided by the manufacturer (R\&D Systems, Inc.) as we previously described [30]. The intra-assay coefficient of variation ranges from 4.1 to 6.5 , and the interassay coefficient of variation ranges from 4.1 to 6.0.

2.4. Determination of 8-epiPGF2a. Serum levels of 8epiPGF2a were determined by means of a competitive ELISA using a commercially available kit (Cayman Chemicals, Ann Arbor, MI USA), as we previously described [31, 32]. This method has a specificity of $100 \%$ for 8 -epiPGF2a, while having minimal crossreactivity with other compounds, mostly 8 isoPGF3a.

2.5. Determination of ox-LDL. Plasma levels of ox-LDL were measured by a competitive enzyme-linked immunosorbent assay using a specific murine monoclonal antibody (4E6) according to the instructions provided by the manufacturer (Mercodia, Uppsala, Sweden) as we previously described [32]. Intra- and interassay coefficients of variation were $6.0 \%$ and $7.0 \%$, respectively.

2.6. Determination of IL-6 and TNF- $\alpha$. Serum IL-6 and TNF$\alpha$ were measured by a high-sensitivity ELISA (Quantikine HS human IL-6 and TNF- $\alpha$, Research \& Diagnostic Systems Europe Ltd., Abington UK). The sensitivity of the ELISA system was less than $0.5 \mathrm{pg} / \mathrm{ml}$ for both IL- 6 and TNF- $\alpha$.

2.7. Determination of hs-CRP, sICAM-1, and sVCAM-1. Serum hs-CRP levels were measured by high-sensitivity immunoturbidimetry (Cobas Integra 800, Roche). Serum levels of the molecules sICAM-1 and sVCAM-1 were measured by a sandwich enzyme immunoassay technique (ELISA) using commercially available standard kits (Quantikine human sICAM-1 and VCAM-1, Research \& Diagnostic Systems Europe Ltd., Abington UK). The sensitivity of the ELISA system was less than $2 \mathrm{ng} / \mathrm{ml}$ for both sICAM-1 and sVCAM-1.

2.8. Echocardiography. Left ventricular mass (LVM) was assessed by $2 \mathrm{D}$-mode echocardiogram usually within one week and no longer than one month from study entry, by a single cardiologist who followed a predefined protocol for the recordings and measurements and was blinded to the clinical and biochemical data. LV mass was estimated with Devereux formula, and LV mass index (LVMI) was calculated by dividing LV mass with patient's BMI $\left[\mathrm{LVMI}=\mathrm{LV}\right.$ mass $\left.(\mathrm{g}) / \mathrm{BSA}\left(\mathrm{m}^{2}\right)\right][33,34]$. Left ventricle ejection fraction (LVEF) and left ventricle shortening fraction (LVFS) were estimated as well.

\section{Statistical Analysis}

Frequencies and percentages were used to describe all categorical data collected, while means with standard deviation (normally distributed data) and medians with interquartile range (IQ, nonnormally distributed data) for the scale measurements. Pearson's correlation coefficient or Spearman's rho, depending on the data distribution, was used to assess linear relationships, while differences in the PCSK9 levels between dichotomous data were examined with the independent samples $t$-test, after checking the normality assumption under the Kolmogorov-Smirnov test. Multiple regression analysis was carried out to test the effect of all independent parameters for their effect on plasma PCSK9 levels. Included parameters were all with a $p<0.2$ in the univariate analysis (sex, diabetes mellitus, statin treatment, $\log$ UPR, Lp(a), TG, CRP, fibrinogen, ox-LDL, and sICAM-1). The level of significance was set at 0.05 , and the analysis was conducted using the SPSS v23.0 software.

\section{Results}

Patients' mean age was 66 years, and 51 (55.4\%) were males. Demographic, clinical, and laboratory data and comorbidities of the 92 patients are shown in Table 1 . The mean eGFR was $47.3 \pm 25.7 \mathrm{mg} / \mathrm{ml} / 1.73 \mathrm{~m}^{2}$, and the median proteinuria was $323 \mathrm{mg} / 24 \mathrm{~h}$ (IQR, $140-1148 \mathrm{mg} / 24 \mathrm{~h}$ ).

The mean PCSK9 plasma level in CKD patients was $278.10 \mathrm{ng} / \mathrm{ml}$ in comparison with significantly lower PCSK9 level in 20 control subjects (mean value $156 \pm 43 \mathrm{ng} / \mathrm{ml}$ ) (Table 2). Median hs-CRP was $1.0(0.3-4.3) \mathrm{mg} / \mathrm{l}$, mean fibrinogen was $433 \mathrm{mg} / \mathrm{dl}$, median IL-6 was 3.1 (1.9-4.5) $\mathrm{pg} / \mathrm{ml}$, median TNF- $\alpha$ was 1.9 (1.4-3.0) $\mathrm{pg} / \mathrm{ml}$, mean 8epiPGF2a was $110 \mathrm{pg} / \mathrm{ml}$, ox-LDL was $79.1 \mathrm{U} / \mathrm{l}$, median sICAM-1 was 240 (200-317) $\mathrm{ng} / \mathrm{ml}$, and mean VCAM-1 was $917.3 \mathrm{ng} / \mathrm{ml}$.

4.1. Associations of Kidney Function Parameters. Age, systolic BP, UPR, uric acid, PTH, and LVMI increased with decreasing eGFR $(p<0.05$ for age and $p<0.001$ for all other 
TABle 1: Demographic characteristics, clinical and laboratory data, and comorbidities of the 92 CKD patients and the 20 controls.

\begin{tabular}{lcc}
\hline Parameters & $\begin{array}{c}\text { CKD patients } \\
(n=92)\end{array}$ & $\begin{array}{c}\text { Controls } \\
{[20]}\end{array}$ \\
\hline Age (years) & $65.8 \pm 12.45$ & $35 \pm 6$ \\
Gender (male), $n \%$ & $51(55.4 \%)$ & $6(30 \%)$ \\
BMI (kg/m $\left.{ }^{2}\right)$ & $47.29 \pm 25.68$ & $22.7 \pm 2.0$ \\
SBP (mmHg) & $142 \pm 19$ & $111 \pm 12$ \\
DPB (mmHg) & $81 \pm 11$ & $67 \pm 8$ \\
Hypertension, $n \%$ & $80(74 \%)$ & 0 \\
Diabetes mellitus, $n \%$ & $26(28.3 \%)$ & 0 \\
History of CAD, $n \%$ & $25(27 \%)$ & 0 \\
Statin treatment, $n \%$ & $33(36 \%)$ & 0 \\
eGFR/CKD-EPI (ml/min/1.73 & $47.3 \pm 25.7$ & $89.5 \pm 18.3$ \\
m $\left.{ }^{2}\right)$ & $323(140,1148)$ & - \\
UPR (mg/24h) & $209 \pm 47$ & $172 \pm 27$ \\
T-Chol (mg/dl) & $163 \pm 88$ & $67 \pm 32$ \\
TG (mg/dl) & $52 \pm 15$ & $57 \pm 11$ \\
HDL (mg/dl) & $124 \pm 39$ & $102 \pm 21$ \\
LDL (mg/dl) & $11.1(8.0,29.3)$ & $8(1-24)$ \\
Lp(a) (mg/dl) & $139 \pm 30$ & $159 \pm 23$ \\
APO-Al (mg/dl) & $93 \pm 28$ & $65 \pm 15$ \\
APO-B (mg/dl) & $4.23 \pm 0.40$ & $4.4 \pm 0.30$ \\
Albumin (mg/dl) & $6.82 \pm 1.64$ & $5.1 \pm 1.35$ \\
Uric acid (mg/dl) & $74(47,121)$ & - \\
PTH pg/ml & $13.0 \pm 1.6$ & $13.6 \pm 1.4$ \\
Hb (g/dl) & $6.1(5.7,7.2)$ & - \\
HbAlc (\%) & $135.7 \pm 47.1$ & - \\
LVMI (g/m $\left.{ }^{2}\right)$ & $69 \pm 10$ & - \\
EF (\%) & $37 \pm 8$ & - \\
FS (\%) & & \\
\hline
\end{tabular}

TABLE 2: Levels of PCSK9, inflammation, oxidative stress, and endothelial dysfunction markers in the $92 \mathrm{CKD}$ patients and the 20 controls.

\begin{tabular}{lcc}
\hline PCSK9 $(\mathrm{ng} / \mathrm{ml})$ & $278.10 \pm 80.2$ & $156.2 \pm 43.1$ \\
CRP $(\mathrm{mg} / \mathrm{l})$ & $1.0(0.3,4.3)$ & $1.0(1.0,2.2)$ \\
Fibrinogen $(\mathrm{mg} / \mathrm{dl})$ & $433 \pm 174$ & $312 \pm 136$ \\
IL-6 $(\mathrm{pg} / \mathrm{ml})$ & $3.1(1.9,4.5)$ & $0.8(0.5,2.6)$ \\
TNF- $\alpha(\mathrm{pg} / \mathrm{ml})$ & $1.9(1.4,3.0)$ & $0.7(0.1,3.3)$ \\
8-epiPGF2a $(\mathrm{pg} / \mathrm{ml})$ & $110(92,138)$ & $45 \pm 19$ \\
ox-LDL $(\mathrm{U} / \mathrm{l})$ & $79.1 \pm 23.9$ & $42 \pm 15$ \\
sICAM-1 $(\mathrm{ng} / \mathrm{ml})$ & $240(200,317)$ & $224 \pm 20$ \\
sVCAM-1 $(\mathrm{ng} / \mathrm{ml})$ & $917.3 \pm 377.1$ & $602.9 \pm 145$ \\
\hline
\end{tabular}

parameters), whereas BMI and hemoglobin decreased with advanced CKD $(p<0.001$ for both). Among lipid profile markers, only APO-A1 was significantly associated with eGFR in a direct fashion $(p=0.001)$. Kidney function expressed as eGFR showed significant inverse association with fibrinogen $(p<0.001)$, IL-6 $(p<0.05)$, TNF- $\alpha$ $(p<0.001), \quad 8$-epiPGF2a $\quad(p<0.001), \quad$ and sVCAM-1 $(p<0.001)$.

Urine protein daily excretion was strongly associated (in a direct fashion) with TG $(p<0.001)$, LVMI $(p<0.001)$, 8 epiPGF2a $(p=0.007)$, and sVCAM-1 $(p<0.001)$.

We did not find a significant association between PCSK9 levels and markers of the kidney function in the nondialysis CKD population of our study.

4.2. Associations between Inflammation, Oxidative Stress, and Endothelial Dysfunction Markers. Associations between inflammation, oxidative stress, and endothelial dysfunction markers are shown in Table 3. High-sensitivity CRP was found to have a direct association with fibrinogen $(p<0.001)$, IL-6 $(p<0.001), 8$-epiPGF2a $(p<0.001)$, and sICAM-1 $(p<0.001)$. Interleukin-6 significantly associated in a direct manner with fibrinogen $(p<0.001)$, TNF- $\alpha$ $(p=0.001)$, and sVCAM-1 $(p<0.001)$, while TNF- $\alpha$ and sVCAM-1 had a direct strong association $(p<0.001)$. sVCAM-1 was found to correlate directly with sICAM-1 as well $(p<0.001)$. Significant associations of oxidative stress markers were direct association of 8-epiPGF2a with hsCRP, sICAM- 1 , and sVCAM- 1 ( $p<0.001, p=0.001$, and $p$ $=0.003$, respectively), while ox-LDL associated directly with lipid metabolism parameters, TG, T-Chol, LDL-C, and APOB $(p<0.001$ for all $)$ and with $\operatorname{Lp}(\mathrm{a})(p=0.02)$.

4.3. Associations of PCSK9 with Lipid Metabolism Parameters, Inflammation, Oxidative Stress, and Endothelial Dysfunction Markers. PCSK9 levels were directly associated with TG $(p=0.03), \operatorname{Lp}(\mathrm{a})(p=0.01)$, and ICAM-1 $(p=0.03)$ (Figure 1). There was a significant positive correlation between PCSK9 levels and statin treatment. Patients on statin treatment had higher PCSK9 levels in comparison with those who did not $(318.19 \mathrm{ng} / \mathrm{ml}$ vs. $253.57 \mathrm{ng} / \mathrm{ml}, p<0.001)$. In our studied CKD population of stages II-IV, PCSK9 plasma levels were not associated with kidney function parameters, diabetes mellitus, and cardiovascular comorbidity or with echocardiography indices.

Multiple regression analysis, as described above, was conducted to test all independent parameters for their effect on plasma PCSK9 levels. The results showed a statistically significant effect of the $\mathrm{Lp}(\mathrm{a})$ values, as well as of statin intake. Specifically, for each unit of higher $\operatorname{Lp}(\mathrm{a})$, an increase by 3.082 is expected for the PCSK9 values (95\% CI: $0.935-$ $5.228, p=0.006)$. At the same time, patients receiving statins are expected to have on average $63.8 \mathrm{ng} / \mathrm{ml}$ higher PCSK9 values compared to patients not receiving statins (95\% CI: $14.6-113.5, p=0.012$ ).

\section{Discussion}

This study shows that in nondialysis CKD patients, plasma levels of PCSK9 are directly associated with the endothelial dysfunction biomarker sICAM-1 and with Lp(a), an established risk factor for myocardial infraction and cardiovascular death in CKD patients [35]. A plethora of experimental 
TABle 3: Associations between inflammation, oxidative stress, endothelial dysfunction, and lipid metabolism markers in the CKD patients.

\begin{tabular}{lccc}
\hline & & Spearman rho & $p$ value \\
\hline \multirow{4}{*}{ hs-CRP } & Fibrinogen & 0.44 & $<0.001$ \\
& 8-epiPGF2a & 0.32 & $<0.001$ \\
& IL-6 & 0.27 & $<0.001$ \\
& sICAM-1 & 0.35 & $<0.001$ \\
IL-6 & Fibrinogen & 0.32 & $<0.001$ \\
& TNF- $\alpha$ & 0.25 & 0.001 \\
TNF- $\alpha$ & sVCAM-1 & 0.31 & $<0.001$ \\
sVCAM-1 & sVCAM-1 & 0.35 & $<0.001$ \\
8-epiPGF2a & sICAM-1 & 0.27 & $<0.001$ \\
& sICAM-1 & 0.26 & 0.001 \\
& sVCAM-1 & 0.23 & 0.003 \\
& TG & 0.30 & $<0.001$ \\
ox-LDL & T-Chol & 0.68 & $<0.001$ \\
& LDL-C & 0.71 & $<0.001$ \\
& APO-B & 0.64 & $<0.001$ \\
& Lp(a) & 0.20 & 0.02 \\
\hline
\end{tabular}

and clinical studies have established the central role of PCSK9 in lipid metabolism while robust evidence supports the implication of PCSK9 in the inflammatory nature of atherosclerosis [16].

Inflammation, oxidative stress, and endothelial dysfunction hold a key role in ASCVD in CKD patients. Studies investigating the role of PCSK9 in atherosclerosis beyond LDL-C regulation completely lack in the CKD high-risk population. In this clinical study, for the first time, we tested the possible association of PCSK9 levels with inflammation, oxidative stress, and endothelial dysfunction biomarkers in a cohort of CKD nondialysis patients. We found that sICAM-1 levels were directly associated with PCSK9 levels. The increased levels of sICAM-1 are shown to be an independent predictor of mortality in predialysis patients with cardiovascular disease $[36,37]$. In the basic step of atherosclerotic vascular damage, LDL-C stimulates endothelial cells in the inner layer which express on their surface ICAM-1 and VCAM-1 and further promote the adhesion of circulating inflammatory leucocytes. Serum PCSK9 levels have been evaluated in healthy participants and showed an independent association with arterial stiffness, a wellestablished risk factor for atherosclerotic cardiovascular disease [38]. Moreover, in patients undergoing coronary angiography for acute coronary syndrome or stable angina, PCSK9 was found to linearly associate with the fraction and amount of necrotic core tissue in coronary atherosclerosis, independently of serum LDL cholesterol levels and statin use [39]. In an animal model, administration of lipopolysaccharides in PCSK9 knockout mice diminished the expression of VCAM-1 from endothelial vascular cells compared with wild type [16]. Release of ICAM-1 from vascular endothelial cells was reduced with PCSK9i alirocumab and anti-PCSK9 vaccine AT04 in the APOE $* 3$ Leiden.CETP transgenic mouse model for hyperlipidemia and atherosclerosis $[40,41]$.
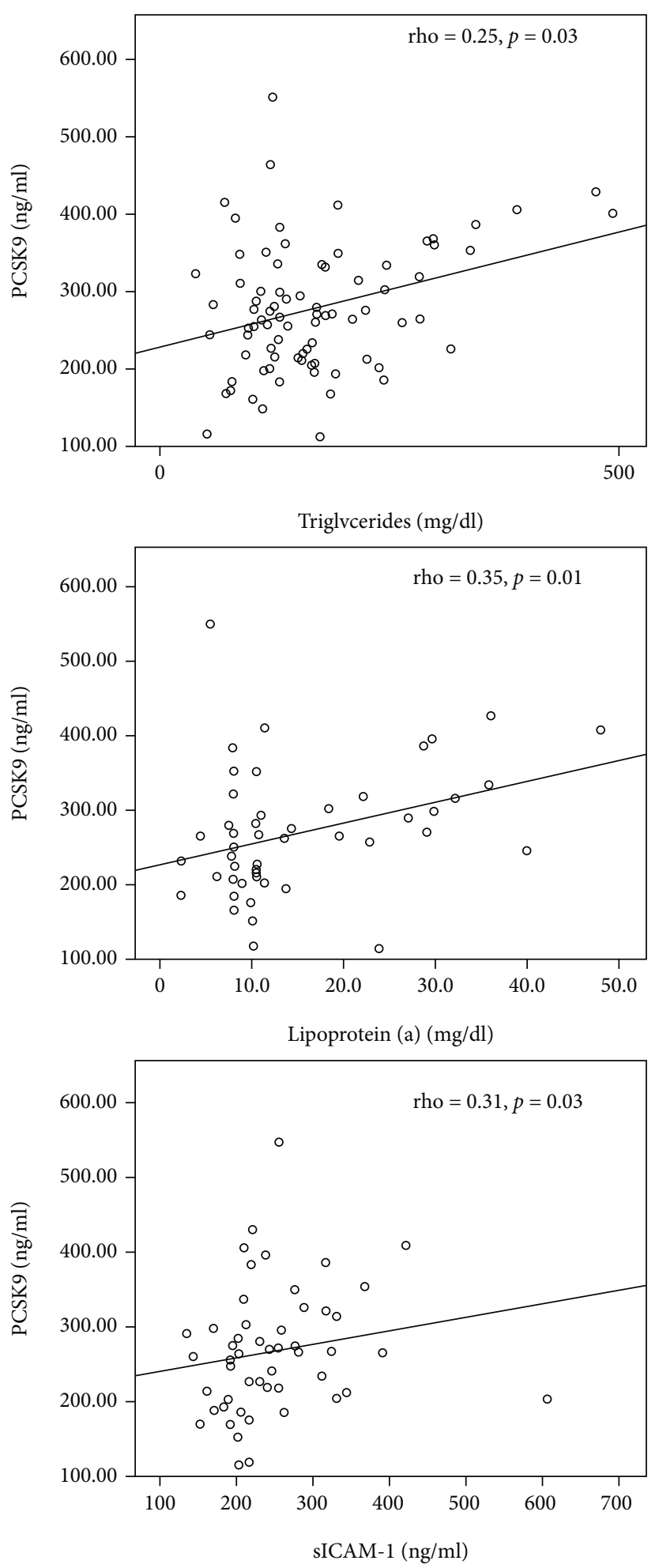

FIgURE 1: Significant associations of PCSK9 with triglycerides, Lp(a), and sICAM-1 in CKD patients.

Among studies estimating PCSK9 levels in nondialysis CKD patients, only two reported the association of a marker of inflammation hs-CRP with PCSK9 levels in this population. In the first one, with 44 CKD patients (eGFR 20.2 $\mathrm{ml} / \mathrm{min} / 1.73 \mathrm{~m}^{2}$ ), the authors reported a rather weak direct 
correlation between the two markers $(r=0.26, p<0.05)$ [42]. In the largest one, Rogacev et al. assessed PCSK9 levels in two cohorts of CKD patients (CARE FOR HOMe cohort and LURIC cohort) and found no correlation between PCSK9 and hs-CRP levels nor with eGFR [13]. In accordance with Rogacev et al., we did not find any correlation between plasma PCSK9 levels and neither hs-CRP nor with kidney function markers, eGFR and UPR, in our patients. Most of the available studies showed no correlation between PCSK9 and eGFR. Interestingly, one small crossover study has shown that plasma PCSK9 can be manipulated in response to therapeutic interventions, which have other hemodynamic benefits such as endothelin antagonism [43]. Moreover, a recent experimental study in a LDL- $\mathrm{R}+/-$ mouse model investigated the potential effect of a vaccine targeting PCSK9 (PCSK9Q $\beta$-003) on hypercholesterolemia and kidney fibrosis. According to their results, vaccination with PCSK9Q $\beta$ 003 had a positive effect on lipid accumulation and renal fibrosis through regulation of fatty acid $\beta$-oxidation [44]. PCSK9 is suggested to be involved in the dyslipidemia and proteinuria of nephrotic syndrome in CKD $[45,46]$. The results of a very recent study of Molina-Jijon et al. showed that the kidney PCSK9 expression was enhanced in the collecting duct of nephrotic patients and animals, supporting the hypothesis that the kidney could be a major source for plasma PCSK9 in nephrotic syndrome. In our study, the vast majority of our patients were not nephrotic [47].

We found that among lipid metabolism parameters, plasma PCSK9 in our CKD patients correlated directly with TG and Lp(a) concentrations. Associations of PCSK9 with lipid profile markers are not consistent in published studies in the different high cardiovascular risk populations [1113]. Dyslipidemia in CKD is characterized mainly by high TG levels and Lp(a), reduced levels of HDL-C, and normal or slightly reduced T-Chol and LDL-C $[10,11]$. Pathogenetically, PCSK9 enhances the degradation of hepatic LDL-R, resulting in an increase in LDL cholesterol levels. We have found no association between plasma PCSK9 levels and TChol, HDL-C, or LDL-C in our patients. Rogacev et al. did not find either, while we both found a direct correlation with TG levels [13]. The absence of an association between PCSK9 and LDL-R might suggest that only a segment of circulating PCSK9 can mediate the degradation of LDL-R in addition to the fact that $36 \%$ of our patients were receiving statins. In our study, patients receiving statin had on average 63.8 $\mathrm{ng} / \mathrm{ml}$ higher PCSK9 values compared to patients not receiving statins. It is known that statins inhibit 3-hydroxy-3methylglutaryl coenzyme A reductase (HMGCoAR), a ratelimiting enzyme in cholesterol biosynthesis, and have been shown to significantly increase PCSK9 mRNA in HepG2 cells and primary human hepatocytes through activation of the sterol regulatory element-binding protein-2 (SREBP-2) pathway [48]. Increased levels of PCSK9 levels in patients on statin treatment could indicate a possible implication of PCSK9 in the lipoprotein TG contents by lipoprotein lipase regulation in CKD patients and an effect on high cardiovascular burden in this patient population. Nevertheless, more dedicated studies are required in order to elucidate the role of PCSK9 along with the role of PCSK9i in CKD.
Lipoprotein (a), a subtype of LDL-C, is an established risk factor for ASCVD in the general and CKD population [11, 49]. The metabolic pathways of $\operatorname{Lp}(\mathrm{a})$ production and clearance are not completely elucidated yet. Nevertheless, robust clinical evidence showed that PCSK9i reduced levels of $\mathrm{Lp}$ (a) by $20-30 \%$ and contributed to reduction of incident major cardiovascular event. Proposed mechanisms are either by increasing catabolism or by reducing production $[49,50]$. In accordance to our results, a direct association between PCSK9 levels and Lp(a) was found by Bermudez-Lopez et al. in a cross-sectional study including 209 nondiabetic CKD patients not receiving statin treatment [51].

In this study, in line with our previous publications, we found significant associations between severity of CKD and inflammation (IL-6, TNF- $\alpha$ ), oxidative stress (8-epiPGF2), and endothelial dysfunction (fibrinogen, sICAM-1, and sVCAM-1) markers and interesting correlations among these novel biomarkers as part of their well-recognized interplay in the uremic milieu $[52,53]$. The results of this study did not demonstrate any association between PCSK9 with the majority of these biomarkers. On the other hand, a growing body of experimental evidence, as aforementioned, highlights the key role of PCSK9 in the pathogenesis of atherosclerosis by its implication in inflammation, apoptosis, oxidative stress, and endothelial damage [16, 54]. Clinical observational data are conflicting regarding the role of PCSK9 as a predictive risk factor for mortality in CKD patients. In a recent prospective study, Strålberg et al. included 265 patients starting dialysis and found a $U$ shape association of PCSK9 levels with all-cause mortality independently of a number of confounders [55]. In contrast to this study, Rogacev et al. failed to demonstrate PCSK9 as a prognostic risk factor for cardiovascular outcomes in nondialysis CKD patients [13].

Our study has some strengths and limitations. To our knowledge, this is the only clinical observational study examining the possible association between PCSK9 with a panel of inflammation, oxidative stress, and endothelial dysfunction markers in nondialysis CKD patients. The main limitations of our study are rather small sample size and crosssectional observational study design. Again, due to the observational nature of our findings, causality cannot be inferred from our data.

\section{Conclusion}

The emerging experimental data indicate that PCSK9 might have additional roles other than regulating blood LDL-C, while PCSK9i have emerged as a very promising category of hypolipidemic agents for the treatment of high-risk populations unable to achieve LDL-C target levels. Clinical data in CKD patients are scarce and not consistent regarding the role of PCSK9 in the inflammatory arm of atherosclerosis and on cardiovascular outcome. In our study, we showed that PCSK9 is possible to be a piece of the complex, unravel puzzle of ASCVD in CKD. As CKD patients remain a population with unmet needs in the management of dyslipidemia and cardiovascular morbidity and mortality, PCSK9 might be an interesting therapeutic target for the treatment of 
atherosclerotic disease beyond LDL-C regulation. Further prospective research is warranted to elucidate the effects of PCSK9 and PCSK9i in patients with reduced renal function.

\section{Data Availability}

The demographic, clinical, and laboratory data of the patients used to support the findings of this study are available from the corresponding author upon request. In any case, personal data protection will be ensured.

\section{Conflicts of Interest}

The authors declare that they have no conflicts of interest.

\section{References}

[1] M. Tonelli, P. Muntner, A. Lloyd et al., "Risk of coronary events in people with chronic kidney disease compared with those with diabetes: a population-level cohort study," Lancet (London, England), vol. 380, no. 9844, pp. 807-814, 2012.

[2] A. S. Go, G. M. Chertow, D. Fan, C. E. McCulloch, and C. Y. Hsu, "Chronic kidney disease and the risks of death, cardiovascular events, and hospitalization," The New England Journal of Medicine, vol. 351, no. 13, pp. 1296-1305, 2004.

[3] E. L. Schiffrin, M. L. Lipman, and J. F. E. Mann, "Chronic kidney disease: effects on the cardiovascular system," Circulation, vol. 116, no. 1, pp. 85-97, 2007.

[4] P. Libby, P. M. Ridker, and A. Maseri, "Inflammation and atherosclerosis," Circulation, vol. 105, no. 9, pp. 1135-1143, 2002.

[5] D. Steinberg, "Atherogenesis in perspective: hypercholesterolemia and inflammation as partners in crime," Nature Medicine, vol. 8, no. 11, pp. 1211-1217, 2002.

[6] V. Cachofeiro, M. Goicochea, S. G. de Vinuesa, P. Oubiña, V. Lahera, and J. Luño, "Oxidative stress and inflammation, a link between chronic kidney disease and cardiovascular disease," Kidney International. Supplement, vol. 111, pp. S4-S9, 2008.

[7] P. Stenvinkel, J. J. Carrero, J. Axelsson, B. Lindholm, O. Heimbürger, and Z. Massy, "Emerging biomarkers for evaluating cardiovascular risk in the chronic kidney disease patient: how do new pieces fit into the uremic puzzle?," Clinical Journal of the American Society of Nephrology, vol. 3, no. 2, pp. 505-521, 2008.

[8] C. Zoccali, "Traditional and emerging cardiovascular and renal risk factors: an epidemiologic perspective," Kidney International, vol. 70, no. 1, pp. 26-33, 2006.

[9] A. Duni, V. Liakopoulos, K.-P. Rapsomanikis, and E. Dounousi, "Chronic kidney disease and disproportionally increased cardiovascular damage: does oxidative stress explain the burden?," Oxidative Medicine and Cellular Longevity, vol. 2017, 15 pages, 2017.

[10] V. Tsimihodimos, E. Dounousi, and K. C. Siamopoulos, "Dyslipidemia in chronic kidney disease: an approach to pathogenesis and treatment," American Journal of Nephrology, vol. 28, no. 6, pp. 958-973, 2008.

[11] C. J. Ferro, P. B. Mark, M. Kanbay et al., "Lipid management in patients with chronic kidney disease," Nature Reviews. Nephrology, vol. 14, no. 12, pp. 727-749, 2018.
[12] M. Farnier, "PCSK9 : de la decouverte aux applications therapeutiques," Archives of Cardiovascular Diseases, vol. 107, no. 1, pp. 58-66, 2014.

[13] K. S. Rogacev, G. H. Heine, G. Silbernagel et al., "PCSK9 plasma concentrations are independent of GFR and do not predict cardiovascular events in patients with Decreased GFR," PLoS One, vol. 11, no. 1, article e0146920, 2016.

[14] G. G. Schwartz, P. G. Steg, M. Szarek et al., "Alirocumab and cardiovascular outcomes after acute coronary syndrome," The New England Journal of Medicine, vol. 379, no. 22, pp. 2097-2107, 2018.

[15] P. P. Toth, J. P. Dwyer, C. P. Cannon et al., "Efficacy and safety of lipid lowering by alirocumab in chronic kidney disease," Kidney International, vol. 93, no. 6, pp. 1397-1408, 2018.

[16] Z.-H. Tang, T.-H. Li, J. Peng et al., "PCSK9: a novel inflammation modulator in atherosclerosis?," Journal of Cellular Physiology, vol. 234, no. 3, pp. 2345-2355, 2019.

[17] M. Ruscica, Ricci, C. Macchi et al., "TNF-alpha induces proprotein convertase subtilisin kexin type 9 (PCSK9) expression in hepatic HepG2 cell line in a SOCS-3-dependent manner," Atherosclerosis, vol. 252, pp. e197-e198, 2016.

[18] E. A. Stein, S. Mellis, G. D. Yancopoulos et al., "Effect of a monoclonal antibody to PCSK9 on LDL cholesterol," The New England Journal of Medicine, vol. 366, no. 12, pp. 11081118, 2012.

[19] Z.-H. Tang, J. Peng, Z. Ren et al., "New role of PCSK9 in atherosclerotic inflammation promotion involving the TLR4/NF$\kappa \mathrm{B}$ pathway," Atherosclerosis, vol. 262, pp. 113-122, 2017.

[20] Z. Tang, L. Jiang, J. Peng et al., "PCSK9 siRNA suppresses the inflammatory response induced by oxLDL through inhibition of NF- $\kappa \mathrm{B}$ activation in THP-1-derived macrophages," International Journal of Molecular Medicine, vol. 30, no. 4, pp. 931938, 2012.

[21] A. Liu and J. Frostegård, "PCSK9 plays a novel immunological role in oxidized LDL-induced dendritic cell maturation and activation of $\mathrm{T}$ cells from human blood and atherosclerotic plaque," Journal of Internal Medicine, vol. 284, no. 2, pp. 193-210, 2018.

[22] J. Li, X. Liang, Y. Wang, Z. Xu, and G. Li, "Investigation of highly expressed PCSK9 in atherosclerotic plaques and oxLDL-induced endothelial cell apoptosis," Molecular Medicine Reports, vol. 16, no. 2, pp. 1817-1825, 2017.

[23] Z. Ding, S. Liu, X. Wang et al., "Cross-talk between LOX-1 and PCSK9 in vascular tissues," Cardiovascular Research, vol. 107, no. 4, pp. 556-567, 2015.

[24] K. R. Walley, K. R. Thain, J. A. Russell et al., "PCSK9 is a critical regulator of the innate immune response and septic shock outcome," Science Translational Medicine, vol. 6, no. 258, article 258ra143, 2014.

[25] J. Rannikko, D. Jacome Sanz, Z. Ortutay et al., "Reduced plasma PCSK9 response in patients with bacteraemia is associated with mortality," Journal of Internal Medicine, vol. 286, no. 5 , pp. 553-561, 2019.

[26] B. Gencer, F. Montecucco, D. Nanchen et al., "Prognostic value of PCSK9 levels in patients with acute coronary syndromes," European Heart Journal, vol. 37, no. 6, pp. 546-553, 2016.

[27] Y. Zhang, C.-G. Zhu, R.-X. Xu et al., "Relation of circulating PCSK9 concentration to fibrinogen in patients with stable coronary artery disease," Journal of Clinical Lipidology, vol. 8, no. 5, pp. 494-500, 2014. 
[28] F. Mach, C. Baigent, A. L. Catapano et al., "2019 ESC/EAS guidelines for the management of dyslipidaemias: lipid modification to reduce cardiovascular risk," European Heart Journal, vol. 41, no. 1, pp. 111-188, 2020.

[29] A. S. Levey, J. Coresh, T. Greene et al., "Expressing the Modification of Diet in Renal Disease Study equation for estimating glomerular filtration rate with standardized serum creatinine values," Clinical Chemistry, vol. 53, no. 4, pp. 766-772, 2007.

[30] T. D. Filippatos, E. Liberopoulos, M. Georgoula, C. C. Tellis, A. D. Tselepis, and M. Elisaf, "Effects of increased body weight and short-term weight loss on serum PCSK9 levels - a prospective pilot study," Archives of Medical Science - Atherosclerotic Diseases, vol. 2, pp. e46-e51, 2017.

[31] E. Dounousi, E. Papavasiliou, A. Makedou et al., "Oxidative stress is progressively enhanced with advancing stages of CKD," American Journal of Kidney Diseases, vol. 48, no. 5, pp. 752-760, 2006.

[32] M. S. Kostapanos, A. T. Spyrou, C. C. Tellis et al., "Ezetimibe treatment lowers indicators of oxidative stress in hypercholesterolemic subjects with high oxidative stress," Lipids, vol. 46, no. 4, pp. 341-348, 2011.

[33] D. J. Sahn, A. DeMaria, J. Kisslo, and A. Weyman, "Recommendations regarding quantitation in M-mode echocardiography: results of a survey of echocardiographic measurements," Circulation, vol. 58, no. 6, pp. 1072-1083, 1978.

[34] R. B. Devereux, D. R. Alonso, E. M. Lutas et al., "Echocardiographic assessment of left ventricular hypertrophy: comparison to necropsy findings," The American Journal of Cardiology, vol. 57, no. 6, pp. 450-458, 1986.

[35] A. Bajaj, S. M. Damrauer, A. H. Anderson et al., "Lipoprotein(a) and risk of myocardial infarction and death in chronic kidney disease: findings from the CRIC study (chronic renal insufficiency cohort)," Arteriosclerosis, Thrombosis, and Vascular Biology, vol. 37, no. 10, pp. 1971-1978, 2017.

[36] P. Stenvinkel, B. Lindholm, M. Heimbürger, and O. Heimbürger, "Elevated serum levels of soluble adhesion molecules predict death in pre-dialysis patients: association with malnutrition, inflammation, and cardiovascular disease," Nephrology Dialysis Transplantation, vol. 15, no. 10, pp. 16241630, 2000.

[37] A. Papayianni, E. Alexopoulos, P. Giamalis et al., "Circulating levels of ICAM-1, VCAM-1, and MCP-1 are increased in haemodialysis patients: association with inflammation, dyslipidaemia, and vascular events," Nephrology Dialysis Transplantation, vol. 17, no. 3, pp. 435-441, 2002.

[38] M. Ruscica, N. Ferri, F. Fogacci et al., "Circulating levels of proprotein convertase subtilisin/kexin type 9 and arterial stiffness in a large population sample: data from the Brisighella Heart Study," Journal of the American Heart Association, vol. 6, no. 5, 2017.

[39] J. M. Cheng, R. M. Oemrawsingh, H. M. Garcia-Garcia et al., "PCSK9 in relation to coronary plaque inflammation: results of the ATHEROREMO- IVUS study," Atherosclerosis, vol. 248, pp. 117-122, 2016.

[40] S. Kühnast, J. W. A. van der Hoorn, E. J. Pieterman et al., "Alirocumab inhibits atherosclerosis, improves the plaque morphology, and enhances the effects of a statin[S]," Journal of Lipid Research, vol. 55, no. 10, pp. 2103-2112, 2014.

[41] C. Landlinger, M. G. Pouwer, C. Juno et al., "The AT04A vaccine against proprotein convertase subtilisin/kexin type 9 reduces total cholesterol, vascular inflammation, and athero- sclerosis in APOE*3Leiden.CETP mice," European Heart Journal, vol. 38, no. 32, pp. 2499-2507, 2017.

[42] M. Konarzewski, M. Szolkiewicz, E. Sucajtys-Szulc et al., "Elevated circulating PCSK-9 concentration in renal failure patients is corrected by renal replacement therapy," American Journal of Nephrology, vol. 40, no. 2, pp. 157-163, 2014.

[43] T. E. Farrah, A. Anand, P. J. Gallacher et al., "Endothelin receptor antagonism improves lipid profiles and lowers PCSK9 (proprotein convertase subtilisin/kexin type 9) in patients with chronic kidney disease," Hypertension, vol. 74, no. 2, pp. 323330, 2019.

[44] D. Wu, Y. Zhou, Y. Pan et al., "Vaccine against PCSK9 improved renal fibrosis by regulating fatty acid $\beta$-oxidation," Journal of the American Heart Association, vol. 9, no. 1, article e014358, 2020.

[45] E. Dounousi, S. Papanikolaou, K. Tellis et al., "FP372PCSK9 and Indices of Cardiovascular Morbidity in Patients with Chronic Kidney Disease," Nephrology Dialysis Transplantation, vol. 34, Supplement_1, 2019.

[46] P. Pavlakou, E. Liberopoulos, E. Dounousi, and M. Elisaf, "PCSK9 in chronic kidney disease," International Urology and Nephrology, vol. 49, no. 6, pp. 1015-1024, 2017.

[47] E. Molina-Jijon, S. Gambut, C. Macé, C. Avila-Casado, and L. C. Clement, "Secretion of the epithelial sodium channel chaperone PCSK9 from the cortical collecting duct links sodium retention with hypercholesterolemia in nephrotic syndrome," Kidney International, vol. 98, no. 6, pp. 1449-1460, 2020.

[48] J. Mayne, T. Dewpura, A. Raymond et al., "Plasma PCSK9 levels are significantly modified by statins and fibrates in humans," Lipids in Health and Disease, vol. 7, no. 1, p. 22, 2008.

[49] E. Liberopoulos, "Lipoprotein(a) reduction with proprotein convertase subtilisin/kexin type 9 inhibitors: an unsolved mystery," European Journal of Preventive Cardiology, 2020.

[50] G. G. Schwartz, L. Bessac, L. G. Berdan et al., "Effect of alirocumab, a monoclonal antibody to PCSK9, on long-term cardiovascular outcomes following acute coronary syndromes: rationale and design of the ODYSSEY outcomes trial," American Heart Journal, vol. 168, no. 5, pp. 682-689.e1.

[51] M. Bermudez-Lopez, C. Forne, N. Amigo et al., "An in-depth analysis shows a hidden atherogenic lipoprotein profile in non-diabetic chronic kidney disease patients," Expert Opinion on Therapeutic Targets, vol. 23, no. 7, pp. 619-630, 2019.

[52] K. Ioannou, V. S. Stel, E. Dounousi et al., "Inflammation, endothelial dysfunction and increased left ventricular mass in chronic kidney disease (CKD) patients: a longitudinal study," PLoS One, vol. 10, no. 9, article e0138461, 2015.

[53] E. Dounousi, I. Bouba, B. Spoto et al., "A Genetic Biomarker of Oxidative Stress, the Paraoxonase-1 Q192R Gene Variant, Associates with Cardiomyopathy in CKD: A Longitudinal Study," Oxidative Medicine and Cellular Longevity, vol. 2016, Article ID 1507270, 7 pages, 2016.

[54] C. Macchi, M. F. Greco, M. Botta et al., "Leptin, resistin, and proprotein convertase subtilisin/kexin type 9: the role of STAT3," The American Journal of Pathology, vol. 190, no. 11, pp. 2226-2236, 2020.

[55] T. Strålberg, A. Nordenskjöld, Y. Cao, K. Kublickiene, and E. Nilsson, "Proprotein convertase subtilisin/kexin type 9 and mortality in patients starting hemodialysis," European Journal of Clinical Investigation, vol. 49, no. 7, article e13113, 2019. 